\title{
A new Home Robot Positioning System (HRPS) using IR switched multi ultrasonic sensors
}

\author{
Saeed Shiry Ghidary, Takahiro Tani, Toshi Takamori, Motofumi Hattori \\ Dept. of computer and systems engineering, Fac. of Engineering, Kobe University, 657-8501, Japan \\ \{Shiry, Tani, Takamori, Hattori\}@ octopus.cs.kobe-u.ac.jp
}

\begin{abstract}
In this paper we propose a very fast measuring system for the location of a mobile robot in indoor environment. The proposed localization method utilizes ultrasonic and infrared signals simultaneously. The transmitter, which is mounted on the mobile robot, transmits both ultrasonic and infrared signals at the same time. The receivers, which are located at fix points in the ceiling of the room use the received infrared signal as a trigger to measure the time of flight of ultrasonic signal. The location of robot is computed by measuring its distance from three receivers. The heading of the robot is computed by measuring its position in two successive points while the robot moves from one point to the other. The performance and validity of this system are evaluated using one transmitter and six receivers located in a $6 \mathrm{~m} \times 4 \mathrm{~m}$ room. The positioning error is less than $5 \mathrm{~cm}$.
\end{abstract}

\section{INTRODUCTION}

Robot positioning, or the process of a robot finding its position in environment, is a major concern in mobile robot navigation. This process is crucial to a home or service robot because most of robot operations are related to the position. This position must be found easily and repeatedly.

The goal of the Home Robot Positioning System (HPRS) project is to develop a method for a mobile robot, which can find its location inside a known environment with less effort and time. This is actually realized by using a map of the environment and the sensory readings to compute the location of the robot.

Robot positioning can be done in two basic methods: absolute and relative positioning or a combination of them. Relative positioning is usually based on dead reckoning (i.e., monitoring the wheel revolutions to compute the offset from a known starting position). Dead reckoning is simple, inexpensive, and easy to accomplish in real time. The disadvantage of dead reckoning is its unbounded accumulation of errors. Absolute positioning methods usually rely on navigation beacons, active or passive landmarks, map matching, or satellite based navigation signals [1].

When a robot does not know its initial position and orientation, a cost that a robot finds autonomously its position and orientation is very high. GPS (Global Positioning System) is one of the solutions of this problem. But GPS is inaccurate for navigating mobile robots and can be used only outdoors. In [2] an indoor GPS system is introduced which consists of pseudo satellites and modified commercial receivers to sense the relative position and attitude of space vehicle in a laboratory environment. In this system both robot and target vehicle has GPS receivers. Carrier phase measurement of robot and target are used to compute relative position of robot. This system is too complex and expensive for ordinary robotics applications and can not find initial position of the robot by itself. The navigating beacon systems employ optical wave or sound wave. Ultrasonic sensors have been widely used in positioning systems for robots. These systems may be divided in two category; one is the area of map building by acquiring complete information about the unknown environment and the other is the localization of mobile robot by using sensory information about the environment [3]. The measuring equipment in [4] uses ultrasonic waves to measure location and direction of robot. In this system direction of three fixed points should be measured at the same time. For this purpose three speakers radiate ultrasonic wave with different frequencies and three rotating receivers mounted on the robot are controlled to follow the direction by phase compression. In this system reliable positioning can be done only when the mobile robot is inside the triangle because tracking system becomes unstable for outside of the triangle. In [3] another localization system based on ultrasonic waves are introduced. This system utilizes two passive beacons and a single rotating ultrasonic sensor. The passive beacons consist of two cylinders with different diameters and reflect the ultrasonic pulses coming from the sonar sensor mounted on a pan tilt device on the mobile robot. The position and heading angle of robot is determined from geometric parameter set of beacon acquired from sonar scan data. This system can not be used in real time because robot must stop and scan the environment for finding its location. The system in [5] uses six ultrasonic beacons connected to a transmitter controller, which sequences the filtering of the beacons. It also uses an iterated extended Kalman filter to estimate the optimum values of position and heading.

In recent years many other studies have been made to find the robot position by using a CCD camera and landmarks. They place the landmark at prespecified position with respect to the coordinate system, and obtain the image form the camera mounted on the mobile robot. Then they try to find the positional relation between camera and landmark by using image processing techniques [6,7]. These methods use different patterns for landmark (circular, rectangular or linear) and highly depend on camera calibration and image sensitivity and also are computationally expensive.

According to the objective of our research, we have designed a robot positioning system, which has the following properties:

a) It is very fast and works in real time without any need to scan the environment. The measuring time is less than $10 \mathrm{~ms}$. This makes the processing unit free to do its main job, which is to recognize the human action.

b) There is no limitation in the positioning in different parts of the room. 
c) It is possible to find the heading of robot without use of compass or gyroscope.

d) It finds the initial position of robot by itself.

e) It can be implemented economically.

f) In this system we did not use the reflected beam of ultrasonic wave so problems associated with reflected beam are canceled and we can get more reliable data.

g) It can locate multi robots.

In the following sections basic concepts of this method and result of experiments are discussed.

\section{SYSTEM DESCRIPTION}

\section{A. Home robot system}

The proposed localization method is developed for a mobile robot, which would be used as Home robot: A robot that helps the human in different tasks inside the room. It consists of two parts: The Mobile robot and the Host computer. These two parts communicate with each other via wire less LAN. In fact the host computer is the main controller of the system and there is a master slave relationship between host computer and mobile robot. Host computer consists of a powerful PC with digital I/O interface and wireless LAN connection. It collects data from sensors on the mobile robot or other sensors in the environment and after processing the data, orders the mobile robot to do the necessary function. Commands such as "move", "turn", "capture" and "measure" are sent by host computer to the robot and the robot provides different data for the host.

The mobile robot has three wheels: two servo DC derived wheels are fixed at both side of mobile robot and one castor is attached at the front side of it. Four ultrasonic sensors are mounted in the four sides of the mobile robot to detect obstacles around it. This robot is equipped with an ultrasonic and infrared transmitter module, which can transmit a burst of ultrasonic sound and infrared light simultaneously. The frequencies of these signals are $40 \mathrm{kHz}$ and $455 \mathrm{kHz}$ respectively. It also has an on-board computer, which acts as the controller of robot's motion and sensing system, a wire less LAN transceiver and batteries.

\section{B. The environment}

The environment is the laboratory room, which is assumed to have a two-dimensional floor plane and composed of walls and corners. This room also contains different objects like desks, tables, computers and other laboratory objects.

\section{Principle of measurement}

The proposed measuring system basically consist of one transmitter module which is mounted on the mobile robot and some (6 in our system) receiver modules which are installed on the fixed points in the ceiling of the room. The transmitter module consists of ultrasonic and infrared transmitter arrays and can produce a burst of both signals at the same time. The receiver module also consists of ultrasonic and infrared sensors and necessary circuit for detection and amplification of these signals.

When the transmitter module sends a couple of ultrasonic and infrared pulses, the receiver module receives the infrared signal almost at the same time (the time of flight of light is negligible and the delay of detecting circuit is considered as a fix value in calculations). This signal starts a counter at the receiving circuit which, runs at $23.1 \mathrm{kHz}$. This counter will continue to count until the reception of ultrasonic signal which, will stop the counter. The value of this counter is proportional to the time of flight of ultrasonic pulse and can be used to calculate the distance between receiver and transmitter as follow:

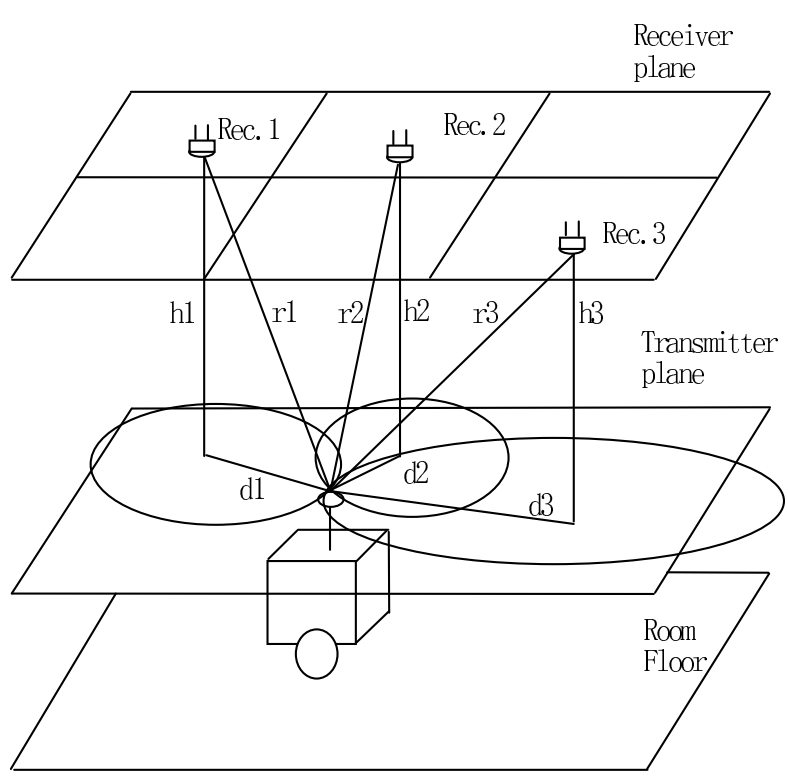

Fig.1. Planes of transmitter and receivers

$$
\begin{aligned}
\mathrm{t} & =\mathrm{n} * \mathrm{f}-\mathrm{t}_{\mathrm{d}} \\
\mathrm{d} & =\mathrm{t} * \mathrm{~V}
\end{aligned}
$$

Where $\mathrm{f}$ is the counter clock rate, $\mathrm{n}$ is the timer count, $t_{d}$ is the delay of detection circuit, $t$ is the total time of flight of sound, $v$ is velocity of sound and $d$ is the range data.

The speed of sound varies slightly with humidity; i.e. max. $0.35 \%$ at $20 \mathrm{c}$ and greatly with temperature; i.e. $7 \%$ variation from $0 \mathrm{c}$ to $40 \mathrm{c}$ [3]. To compensate for inaccuracy caused by temperature, the host computer periodically measures the temperature and corrects the value of sound speed. For measuring the position of the robot, the host computer sends the "measure" command via wireless LAN to the robot and robot produces a couple of ultrasonic and infrared signals. Then the host computer gets the data of receivers and uses them to calculate the position of robot. The location and the number of receivers are designed so that at least 3 receivers will catch these signals. The range data can be read every $10 \mathrm{~ms}$.

As the receivers are fixed at a plane with known heights and also the transmitter is fixed in a known point on the robot, the vertical distance between transmitter and receivers are known in a priori. So the problem of localization of robot can be done in two dimensions. As shown in figure 1, the range (r) to any given receiver is projected to the plane of transmitter (d), which maps out a circular locus of possible positions.

$$
d=\left(r^{2}-h^{2}\right)^{1 / 2}
$$

The interception of two or more of these loci gives the position of the robot. As shown in figure 2, at least three ranges are used in determining position. Analytic geometry can be used to solve simultaneous equations of three circles to find the common point:

$\left(x_{1}-x_{r}\right)^{2}+\left(y_{1}-y_{r}\right)^{2}=d_{1}{ }^{2}$
$\left(x_{2}-x_{r}\right)^{2}+\left(y_{2}-y_{r}\right)^{2}=d_{2}{ }^{2}$
$\left(x_{3}-x_{r}\right)^{2}+\left(y_{3}-y_{r}\right)^{2}=d_{3}{ }^{2}$ 
$\mathrm{x}_{\mathrm{i}}, \mathrm{y}_{\mathrm{i}}(\mathrm{i}=1 \sim 6)$ are the location of sensors in the global 2D coordinate system which are measured exactly and used in the calculations. $d_{i}$ are the radius of circles which are computed from range data as in equation 2 and $\left(\mathrm{x}_{\mathrm{r}}, \mathrm{y}_{\mathrm{r}}\right)$ are computed position of robot.

Due to random errors introduced in range data, they will not all coincide exactly on a single point, hence the least squares method [8] is used to reduce combined differences to a minimum.

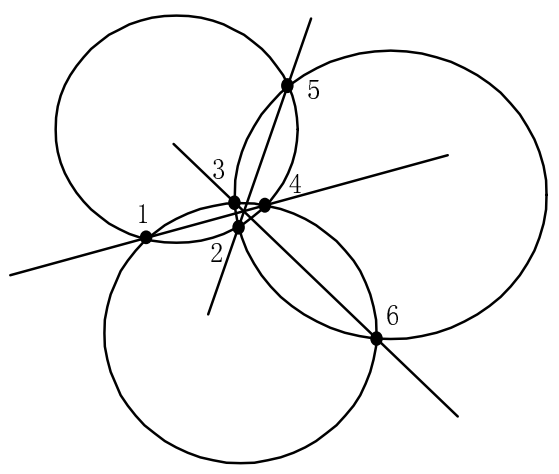

Fig.2. Robot position at the intersection of circles

\section{The receivers and their reception area}

Each receiver module composes of 3 or 4 infrared and ultrasonic sensors. The ultrasonic sensors used in this system have the typical beam pattern given by figure 3 . The distance measurement performed by this kind of sensor is affected by emission/reception angle (figure 4 ) which is about $+/-20$ degree for this sensor. The emission/reception angle is defined as angle of the cone where the intensity/sensitivity is half that on the sensor axis. For infrared sensor the emission angle is about $+/$ 21 and reception angle is about $+/-30$ degree.

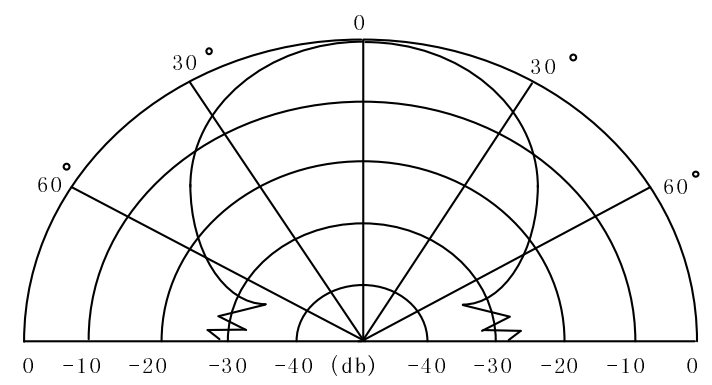

Fig.3. Typical beam pattern at $40 \mathrm{kHz}$.

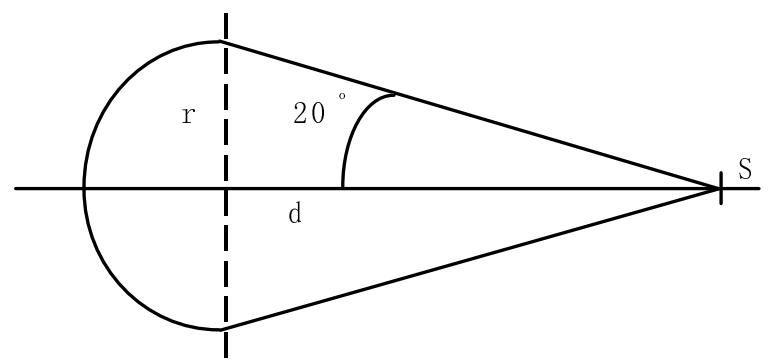

Fig.4. The emission/reception beam
In our system the receiver module is installed in the ceiling of the room in a fixed height. According to the reception angle of this receiver we can define an effective area for it. We define effective area of a receiver, the area inside the plane of transmitter, where the receiver can detect all coming signals sent from this area. This area is the intersection of reception cone of a receiver with the plane of transmitter. We arrange the direction of axes of individual receivers of each receiver module to define effective area for them. Figure 5 shows a sample effective area for a receiver with four sensors.

In this system the room is divided into six equal sized sections (labeled with numbers $1 \sim 6$ in figure 4 ) and one receiver module is installed in each section. Table 1 shows the affective area for each receiver module. The sections covered by each receiver module are chosen so that every section is covered by at least 3 receivers.

\begin{tabular}{|c|c|}
\hline Receiver location & Effective sections \\
\hline 1 & $2,4,5$ \\
\hline 2 & $1,3,4,6$ \\
\hline 3 & $2,5,6$ \\
\hline 4 & $1,2,5$ \\
\hline 5 & $1,3,4,6$ \\
\hline 6 & $2,3,5$ \\
\hline
\end{tabular}

Table 1. Effective area for receiver modules

If we look at table 1 we see that receivers do not cover the section in which they are located. This is because for such sections even a small error in range data would cause a large error in radius of circular loci.

Due to the limitation in the maximum range of receivable signal, which is $4 \mathrm{~m}$ in our system, each receiver can not cover far sections. For example receiver in section 1 can not cover sections 3 or 6 . For smaller rooms it is possible to cover all area of the room with only 4 receivers.

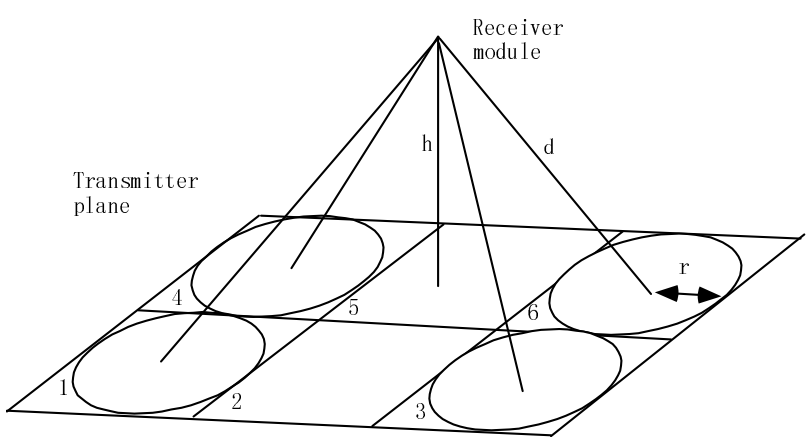

Fig.5. Effective area of a receiver module with 4 receivers

In equation 3 we need three range data to compute the position of the robot. If more than three receivers catch the signal from robot, only three of them would be selected. For selecting 3 out of 4 or 5 possible range data, we first use the three longest range data to estimate the location of the robot. Then according to this estimated location, we choose three sensors, which their data will result in minimum error in the calculations and use them to recompute the exact location of the robot. 


\section{E. The transmitter}

The transmitter module composes of 8 ultrasonic and 8 infrared transmitters arranged at 45 degree intervals with a standing angle of 40 degree (figure 6). This configuration produces an effective transmitting area with total coverage of $5 \times 5 \mathrm{~m}^{2}$.

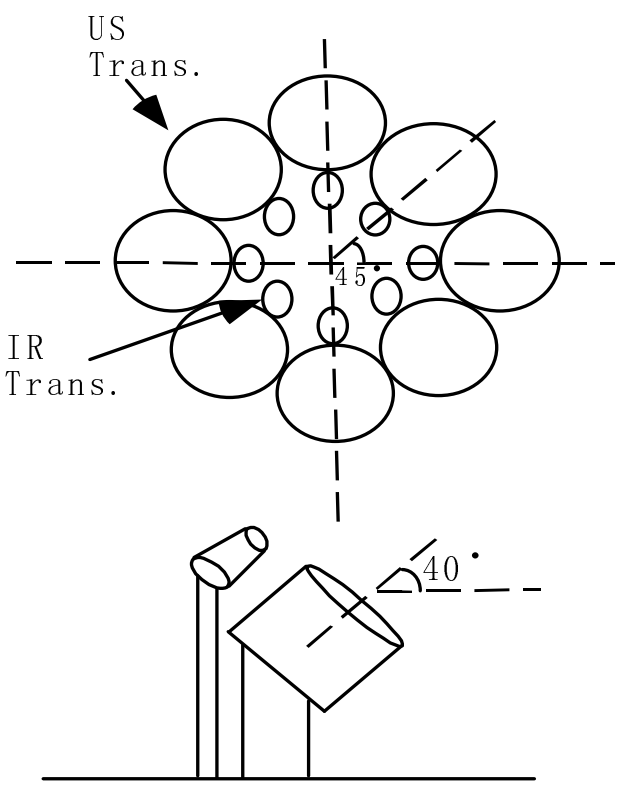

Fig. 6. Arrangement of transducers in transmitter module

\section{EXPERIMENTAL RESULTS AND DISCUSSION}

\section{System setup}

The proposed method was tested using the mobile robot in order to show the effectiveness of this method. The size of indoor environment is $6 \mathrm{~m} \times 4 \mathrm{~m}$. It is divided into six $2 \times 2 \mathrm{~m}^{2}$ area and one receiver module is placed in the center of each area.

The sensors are installed at the heights of $3 \mathrm{~m}$. The transmitter is mounted on the robot in the heights of $80 \mathrm{~cm}$. The 3D positions of receiver modules are measured with exact surveying devices and are used in the software. The receivers are setup to cover their supposed effective area. The system is calibrated by measuring the range from a number of known locations and each receiver is calibrated separately.

The resolution of receiver circuit in measuring the range is 1.5 $\mathrm{cm}$ and it can detect signals coming from a distance up to $4 \mathrm{~m}$.

\section{Static position measurement}

The purpose of this experiment is to evaluate the ability of system in estimating the initial position of the robot. In this experiment robot is located at some known points in the room and its position is measured by this system. Measurement is repeated 10 times for each point. The result of measurement for three sample points is shown in table 2. The first column of this table shows the actual coordinate of each point, which is measured exactly with other surveying methods. The second column is the average of 10 measured data. At column three the absolute value of maximum difference between actual value and measured position is shown. Column four shows the standard deviation of measured data. According to the experiment results the error in positioning is less than $5 \mathrm{~cm}$.

\begin{tabular}{|l|l|l|l|}
\hline $\begin{array}{l}\text { Actual } \\
\text { position } \\
(\mathrm{cm})\end{array}$ & $\begin{array}{l}\text { Mean } \\
\text { measured } \\
\text { Position }\end{array}$ & Max Error & $\begin{array}{l}\text { Standard } \\
\text { deviation }\end{array}$ \\
\hline $\mathrm{X}=250$ & $\mathrm{X}=250.8$ & $\Delta \mathrm{x}=1.5$ & $\operatorname{Std}(\mathrm{x})=0.51$ \\
$\mathrm{Y}=280$ & $\mathrm{Y}=278.8$ & $\Delta \mathrm{y}=3$ & $\operatorname{Std}(\mathrm{y})=1.08$ \\
\hline $\mathrm{X}=430$ & $\mathrm{X}=429.4$ & $\Delta \mathrm{x}=2$ & $\operatorname{Std}(\mathrm{x})=0.78$ \\
$\mathrm{Y}=210$ & $\mathrm{Y}=209.3$ & $\Delta \mathrm{y}=3$ & $\operatorname{Std}(\mathrm{y})=1.18$ \\
\hline $\mathrm{X}=150$ & $\mathrm{X}=148.5$ & $\Delta \mathrm{x}=2.5$ & $\operatorname{Std}(\mathrm{x})=0.78$ \\
$\mathrm{Y}=150$ & $\mathrm{Y}=149.6$ & $\Delta \mathrm{y}=3.5$ & $\operatorname{Std}(\mathrm{y})=1.18$ \\
\hline
\end{tabular}

Table 2. Results of static positioning with 10 readings for each point. (Unit: $\mathrm{cm}$ )

\section{Dynamic position measurement}

The objective of this experiment is to demonstrate the feasibility of the proposed positioning system in real time navigation and path tracking.

This experiment were conducted involving straight motion with speed of $\mathrm{v}=0.3 \mathrm{~m} / \mathrm{sec}$. The robot is given a target position and is wanted to follow the straight path toward the target. Then the path is measured while the robot is doing the job. Figure 7 shows the dynamic estimation results of this experiment, for two different paths. In this figure, the solid lines indicate the reference path and the ' + ' mark indicates the estimated position measured during motion.

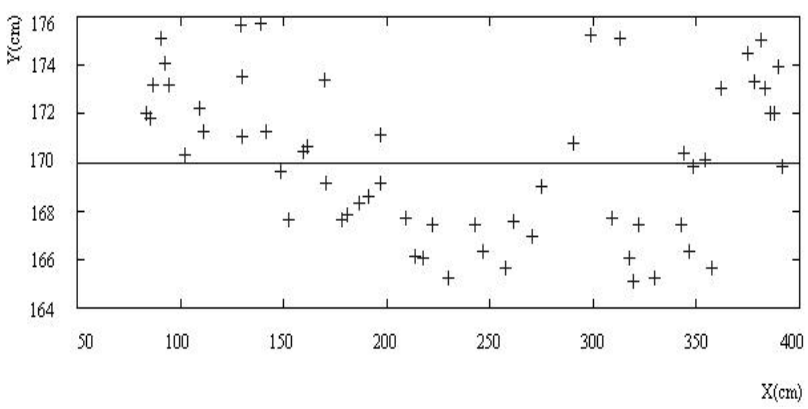

a) Data for motion along $Y=170 \mathrm{~cm}$ straight line.

b)

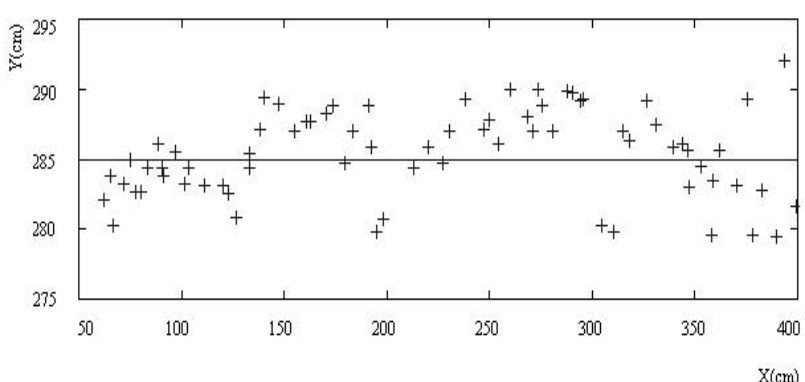

b) Data for motion along $Y=285 \mathrm{~cm}$ straight line.

Fig.7. Experiment result of motion tracking

\section{Estimation of heading angle}

For measuring the heading of the robot, its initial position $\left(x_{0}, y_{0}\right)$ is measured and then the robot is ordered to move to a new point $\left(\mathrm{x}_{1}, \mathrm{y}_{1}\right)$. The heading angle can be calculated as:

$\phi=\tan ^{-1}\left[\left(\mathrm{y}_{1}-\mathrm{y}_{0}\right) /\left(\mathrm{x}_{1}-\mathrm{x}_{0}\right)\right]$ 
Table 3 shows the result of this experiment. The first column shows the actual heading and the other columns show the absolute value of error in measured angle at different moving distances. The experiment results show that the minimum distance required to measure the heading angle is about $50 \mathrm{~cm}$.

\begin{tabular}{|c|c|c|c|c|}
\hline & \multicolumn{4}{|c|}{$\begin{array}{c}\text { Error in measured angle in different } \\
\text { distances (deg.) }\end{array}$} \\
\hline $\begin{array}{l}\text { Actual } \\
\text { heading(deg.) }\end{array}$ & $\begin{array}{c}\text { At } \\
20 \mathrm{~cm}\end{array}$ & $\begin{array}{c}\text { At } \\
30 \mathrm{~cm}\end{array}$ & $\begin{array}{c}\text { At } \\
40 \mathrm{~cm}\end{array}$ & $\begin{array}{c}\text { At } \\
50 \mathrm{~cm}\end{array}$ \\
\hline 0 & 9.8 & 6.6 & 4.9 & 3.2 \\
\hline 90 & 10.1 & 5.9 & 5.2 & 3.1 \\
\hline 45 & 13.4 & 8.8 & 6.9 & 4.1 \\
\hline
\end{tabular}

Table 3. Experiment result of measuring heading angle

\section{Error Discussions}

When implementing the proposed algorithm an error may occur in calculation of the position variable. This error may occur due to the inaccuracy in the range data, which is associated with sensor noise and resolution of receiving circuit. This error can be reduced by increasing the resolution of receiving circuit and also by considering characteristics of range data. It means that the range data, which are too short or too long, or the data, which are out of the limit of the receiving circuit, is removed during the calculations. This will reduce effect of multipath, which is a large source of error in systems based on ultrasonic sensors.

\section{CONCLUSION}

We have developed a home robot localization system, which uses ultrasonic and infrared signals simultaneously to locate the robot in indoor environment. The general structure of the equipment, the algorithm for calculating a location and direction, and the experiments to demonstrate its basic characteristics have been also described.

In this paper we only have discussed the characteristics of the proposed system without considering other possible techniques. For example this system can be used together with dead reckoning data and also estimating algorithms like Kalman filtering which would increase the accuracy of measurements. Also by considering the statistical characteristic of data, we can compensate the error in ultrasonic readings.

\section{REFERENCES}

[1] J. Borenstein and L. Feng, "UMBmark -A Method for Measuring, Comparing, and Correcting Dead-reckoning Errors in Mobile Robots", The University of Michigan, Technical Report UM-MEAM-94-22, December 1994.

[2] Zimmerman, et al. "Experimental Development of an Indoor GPS Based sensing system for Robotic Applications", Navigation, winter 1996-1997,vol 43, No. 4, pp. 375-395.

[3] H.R. Beom and H.S.Cho, "Mobile robot localization using a single rotating sonar and two passive cylindrical beacons" Robotica, 1995, volume 13, pp. 243-252, Cambridge university press.

[4] T. Arai and E. Nakano, "Development of measuring equipment for location and direction (MELODI) using ultrasonic waves", Trans. ASME, Journal of dynamic systems, Measurement and control, 1983, volume 105, pp. 152-156.

[5] L.Kleeman, "Optimal estimation of position and heading for mobile robots using ultrasonic beacons and deadreckoning", IEEE International Conference on Robotics and Automation, Nice, France, pp. 2582-2587, May 10151992.

[6] Jae. H. Kim and Hyung S. Cho, " Real time determination of a mobile robot's position by linear scanning of a landmark", Robotica, 1992, volume 10, pp. 309-319, Cambridge university press.

[7] Kyoung C. Koh, Jae S. Kim and Hyung S. Cho, “ A position estimation system for mobile robots using a monocular image of a 3-D landmark", Robotica, 1994, volume 12, pp. 431-441, Cambridge university press.

[8] R.W.Farebrother, "Linear least squares computations", 1988, Marcel Dekker Inc. 